\title{
EVIDENCE OF TELEPORTED BIOELECTROMAGNETIC ENERGY TRANSFER IN A HUMAN MINIORGAN CAUSING DELAY IN CRYSTALLIZATION
}

\author{
Abrahám A. Embí BS *1凶 \\ ${ }^{* 1}$ MBA, 13442 SW 102 Lane, Miami Florida, USA 33186
}

DOI: https://doi.org/10.29121/granthaalayah.v8.i6.2020.484

Article Type: Research Article

Article Citation: Abrahám A. Embí

BS (2020). EVIDENCE OF

TELEPORTED

BIOELECTROMAGNETIC ENERGY

TRANSFER IN A HUMAN

MINIORGAN CAUSING DELAY IN

CRYSTALLIZATION. International

Journal of Research -

GRANTHAALAYAH, 8(6), 156-162.

https://doi.org/10.29121/granthaa

layah.v8.i6.2020.484

Received Date: 09 May 2020

Accepted Date: 29 June 2020

Keywords:

Hair Bioelectromagmetism

Inter Body Energy Transfer

Hair Shaft

Potassium Ferricyanide

Delay in Crystallization

\begin{abstract}
The purpose of this manuscript is to introduce an optical microscopy methodology documenting an organ inter-tissue parts bioelectromagnetic energy transfer, namely the human hair follicle transferring energy to a short-isolated cut hair shaft segment vertically placed within $\cong 1 \mathrm{~mm}$ distance in front of the hair follicle.

The human hair has two main parts, follicle and shaft and classified as a miniorgan having its own cell divisions and metabolism. Both the follicle and shaft has been independently documented to emit and receive electromagnetic energy. This work shows a previously unrecognized property of the hair shaft, which is acting as an aerial receiver and conduit of Bioelectromagnetic Fields (BEMFs) emitted by the follicle. The received BEMF energy is transient and transferred via teleportation to be immediately discharged by the cut isolated shaft segment. The discharged crystallization imprint mimics the hair follicle proper BEMFs delaying advance of crystallization.
\end{abstract}

\section{INTRODUCTION}

The main purpose of this manuscript is to expand on previous observations of different human tissue or body parts induction of electrobiomagnetic energy [1]. Research has led to classifying the hair follicle as a dynamic miniorgan, having its own "important general molecular and cellular processes beyond skin and hair biology, ranging from organ induction, morphogenesis and regeneration, to pigment and stem cell biology, cell proliferation, migration and apoptosis" [2]. This manuscript introduces biophysical properties of the human hair by documenting an inter-transfer of bioelectromagnetic fields (BEMFs) between the follicle and its shaft.

For the purpose of this presentation the term "Bioelectrobiomagnetic" will be used Why? Because since biomagnetism is defined as the "The existence of weak fluctuating magnetic fields produced by the human body" [3] and these magnetic fields can induce electrical signals are in support of a more specific term, such as Bioelectrobiomagnetic Fields (BEMFs) [4]. In Vitro experiments will show BEMFs emitted by a hair follicle 
transferring onto a hair shaft as evidence of Bioelectromagnetic reception and transmission by the shaft evidenced by a delay in crystallization.

\section{MATERIALS AND METHODS}

\subsection{MATERIALS}

Potassium FerrIcyanide Crystal. K3Fe(CN)6.

CSA \# 13746-66-2.

- Microscope - Glass Slides: 25x75x1mm thickness. Pearl Cat. No. 7101

- Water purity confirmed by hand held electrical fields detector manufactured by Lishtot Detection LTD, Israel. For details link to: https://www.lishtot.com/TDP1.html

- Room relative humidity monitored by an ACU-RITE sensor model \# 01536-RX.

- $\quad$ Digital Video Microscope Celestron II model \# 44341, California, USA.

- Images downloaded to an Apple Computer MacBook Pro Photo Application.

\subsection{METHODS}

\section{The Single Slide Preparation (SSP)}

A single slide preparation (SSP) [5], [6] consists of a single $25 \times 75 \times 1 \mathrm{~mm}$ clean glass slide, where the biological sample is covered with drops of diluted crystals, in this case Potassium Ferricyanide crystals $\left(\mathrm{KFe}_{3}\right)$. All hair samples were placed approximately on the center of a $25 \times 75 \times 1 \mathrm{~mm}$ glass slide. A solution was prepared by using $1 \mathrm{ml}$ of bottled water whose purity was confirmed via a handheld electrical field detector (see materials), then delivered into a test tube where one gram of $\mathrm{KFe}_{3}$ crystals were already placed and manually shaken until the crystals were in solution, a small pipette placed inside. Two drops of the mixed $\mathrm{KFe}_{3}$ (paramagnetic solution) were delivered; care was taken to completely cover tweezers pluck scalp hair and isolated cut shaft segment. Using a wooden toothpick, previously cut short shaft segment was then vertically positioned at approximately $1 \mathrm{~mm}$ distance from the hair follicle. The SSP preparation was then allowed to evaporate. After a complete and total evaporation lasting approximately 45 minutes, the SSP was then viewed microscopically in the normal mode (no filters) at X10 and/or 40X magnification with a video microscope (Celestron. LCD Digital Microscope II model \#44341 Torrance California USA). Still microphotographs and video recordings were downloaded and labeled by using an Apple Inc. iPhoto 8.1.2, App.

\section{RESULTS}

\subsection{BIOELECTROMAGNETISM AND CRYSTALLIZATION}

Several observations were demonstrated by the in vitro experiments, such as:

Inhibiton of Bioelectromagnetic Waves are we witnessing BEMFs teleportation?

- Since the hair follicle and isolated shaft are imbedded in a liquid solution undergoing evaporation, this author expected to see crystallization patterns compatible with BEMFs. This did not occur (See Figure 1). The inhibition of concentric bioelectromagnetic waves emitted by the hair follicle commonly observed in a SSP which are outlined by dense $\mathrm{KFe}_{3}$ crystallization. This inhibition is highlighted by $\mathrm{X}$ as shown in (Fig 1). Refer to Figure 3 below for comparison. Since the BEMFs did not induce crystallization between the follicle and the isolated shaft segment, then the question arises: Are we demonstrating BEMFs teleportation? 


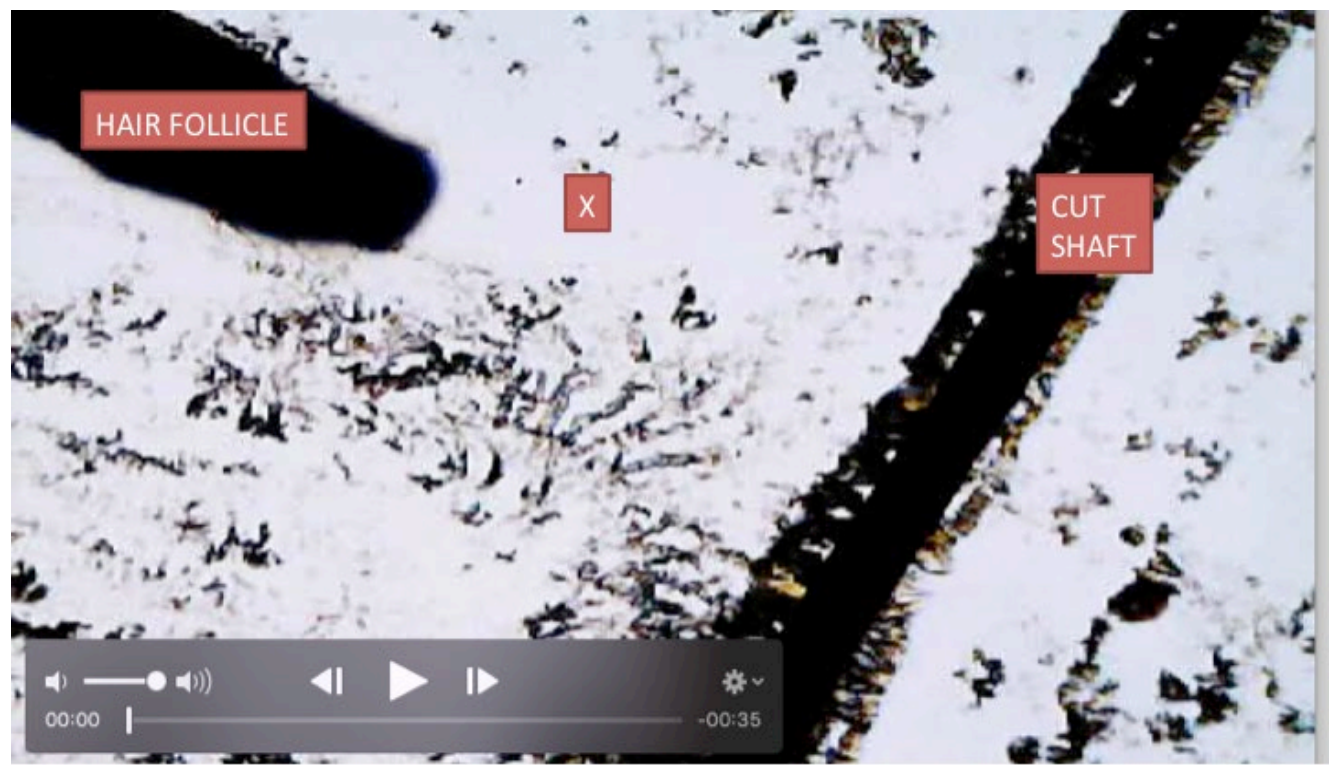

Figure 1: Microphotograph of video frame showing a short cut hair shaft segment placed in front of hair follicle inhibiting crystallization in aSSP KFe3 after evaporation. X: Denotes absence of periodical biomagnetic trails.

\section{Clashing of Diamagnetic with Paramagnetic Fields Delay Crystallization}

- In a control SSP, the observed compactness of the $\mathrm{KFe}_{3}$ is attributed to the clashing of divergent magnetic waves, a repelling incoming magnetism or diamagnetism from the hair follicle, with the paramagnetism of (attraction towards a magnetic field) $\mathrm{KFe}_{3}$ producing a noticeable back (diamagnetic repulsion) and forth (paramagnetic advance) clashing of forces, please refer to Panel $1+$ video-recordings below.

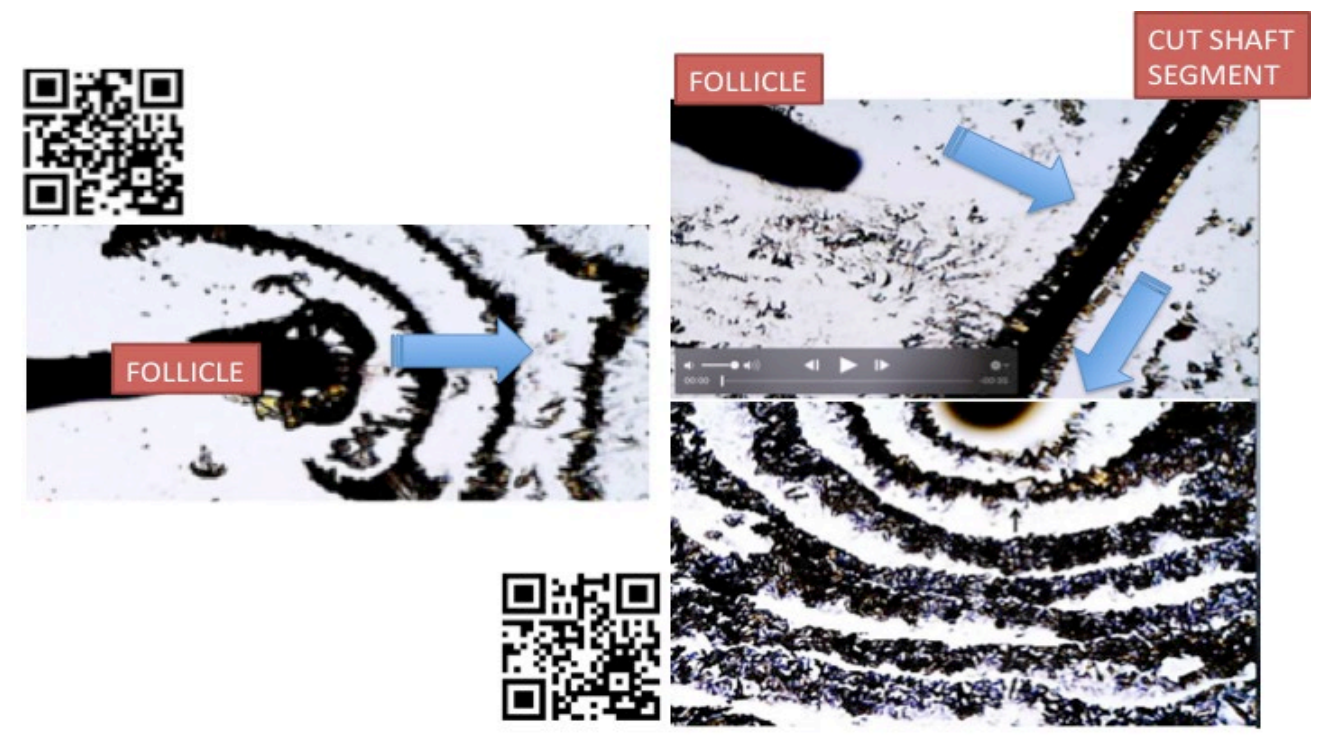

A

B

Panel 1: Blue arrow showing BEMFs flow direction of hair follicle. A: Control hair in SSP $\mathrm{KFe}_{3}$ showing orderly semicircular crystallization from biomagnetic waves. B: Showing a cut hair shaft segment placed in front of the follicle. The follicle is now via teleportation transferring its biomagnetism onto the shaft issue serving as a conduit.

Blue Arrow, panel A showing unimpeded biomagnetic waves progression.

Blue Arrows Panel B, biomagnetic waves now flowing through (cut hair shaft) or alternative conduit.

For additional details Scan QR Codes attached to images.

Panel A evaporation time 3' 29" 
Panel B evaporation time 4'00

\section{The Isolated Hair Shaft Lacks Intrinsic BEMFs}

- When a hair shaft is not under the influence BEMFs, the $\mathrm{KFe}_{3}$ crystals are less compacted, this is attributed to a lack of BEMFs clashing between a diamagnetic and a paramagnetic solution $\left(\mathrm{KFe}_{3}\right)$ as shown in Figure 2 below. This is evidence that the hair shaft by itself lacks the level of metabolic activity necessary to emit BEMFs.

\section{Demonstration of Rapid Crystallization Advance from an Isolated Cut Shaft Segment}

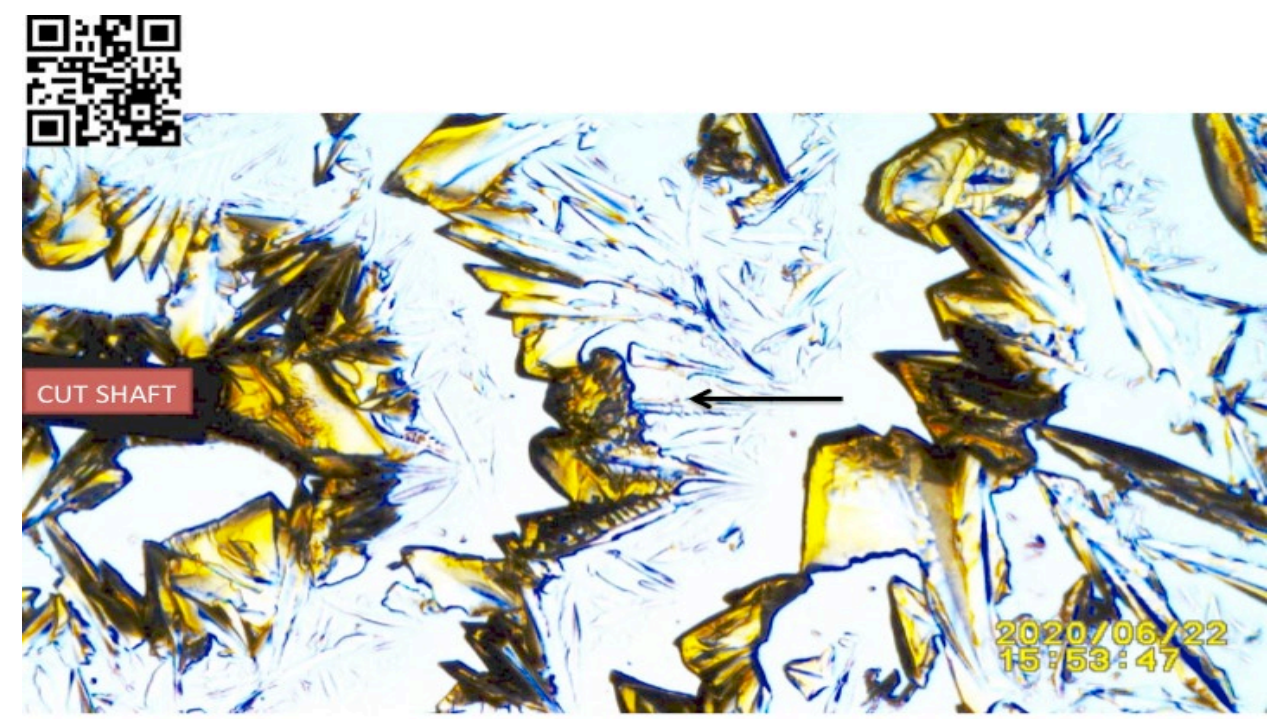

Figure 2: Independent cut hair shaft segment not influenced by divergent bioelectromagentic forces attracting $\mathrm{KFe}_{3}$ crystals.

Black Arrow: Pointing at less compacted $\mathrm{KFe}_{3}$ crystals.

For details link to: https://youtu.be/Yolgu-vFeK0 Or scan QR Code in left upper corner of image.

Evaporation time 1' 40".

\section{Clashing of Hair BEMFs Through Glass Barrier Delays Crystallization}

- When a Ferricyanide or Ferrocyanide solution is within BEMFs reach, the ensuing crystallization is not only delayed; but shows a greater crystals clumping Fig 3 below. The hair follicle BEMFs delay the Ferro or Ferri cyanide crystallization progression for an average of approximately 4 minutes. (Panel 2).

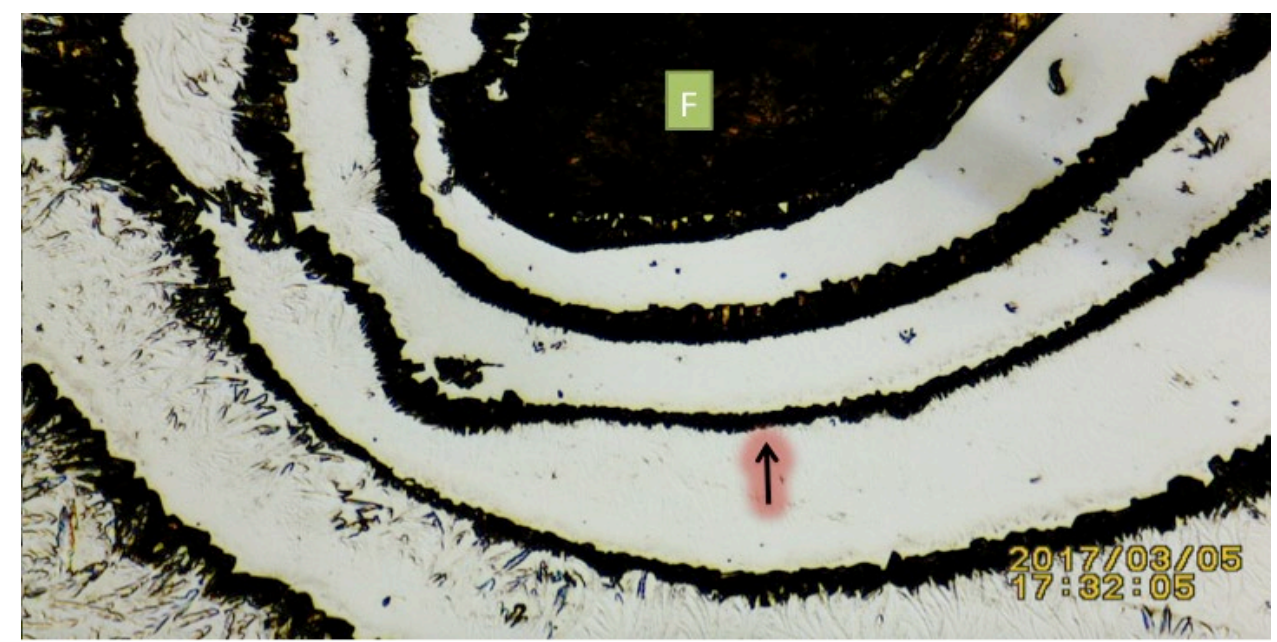

Figure 3: Another example of hair follicle's BEMFs effect on evaporation dynamics and crystals deposition of Potassium Ferrocyanide. F= Follicle

Highlighted Arrow= Heavy crystallization lines. 
Example of a hair follicle BEMFs delaying the crystallization advance of a $\mathrm{KFe}_{2}$ solution through a $1 \mathrm{~mm}$ glass barrier [7].
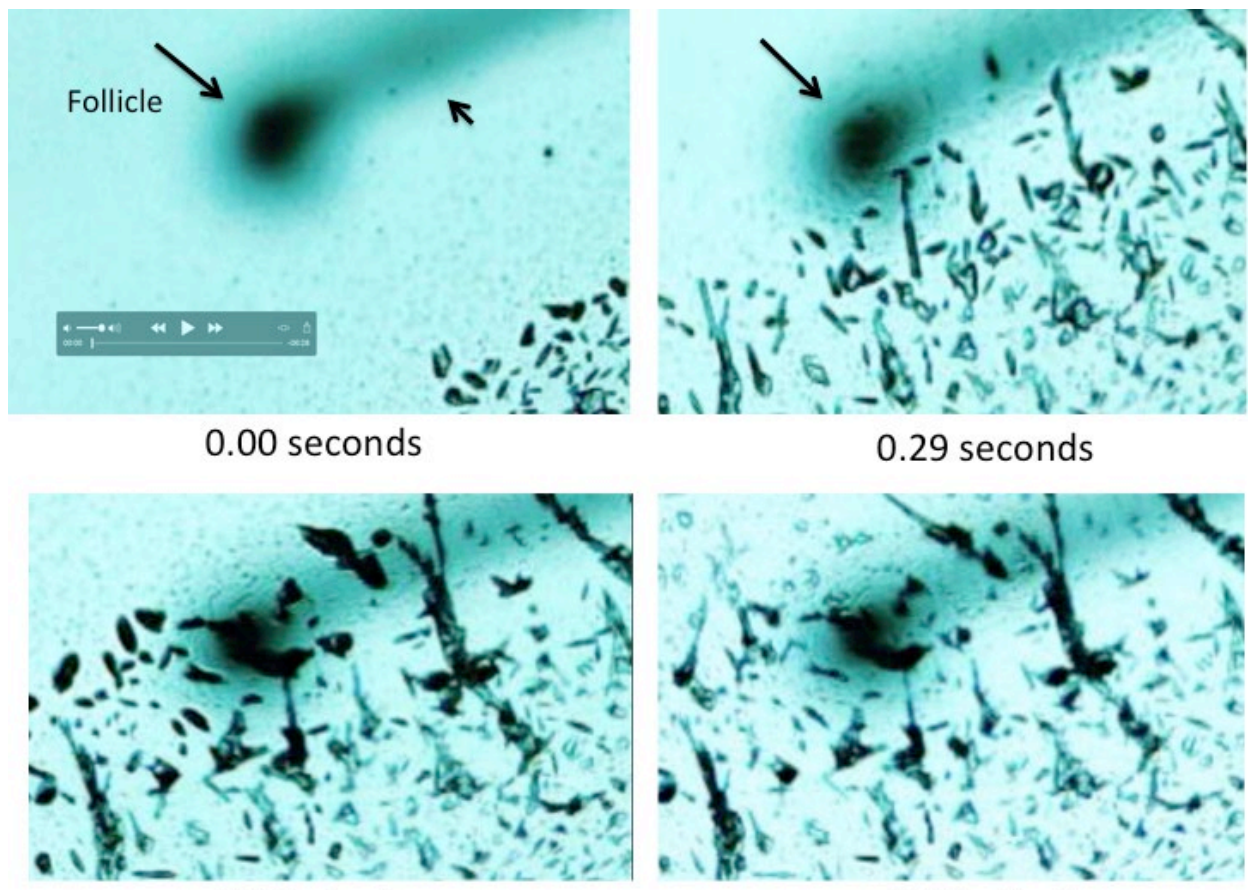

2.29 minutes

4.57 minutes

Panel 2: Notice the rapid advance of the Potassium Ferrocyanide evaporation line during the first 29 seconds. As the evaporation line reaches the hair follice/shaft outline, there is a noticeable delay in progression. This is an example of repulsion caused by the EBMFs effect of a human hair follicle detected by a diamagnetic solution through a $1 \mathrm{~mm}$ glass barrier. For details link to:

https://youtu.be/6iKcEvxY_zE

Or Scan QR Code below.

Panel reproduced from: Cite This Article: Abraham A. Embi Bs. (2018). "THE HUMAN HAIR FOLLICLE PULSATING BIOMAGNETIC FIELD REACH AS MEASURED BY CRYSTALS ACCRETION.” International Journal of Research - Granthaalayah, 6(7), 290-299. https://doi.org/10.5281/zenodo.1341349.

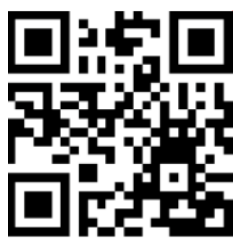

\section{DISCUSSION}

- The human hair has two main anatomical components (the follicle and shaft) and classified as a miniorgan; and herein demonstrated in two different experiments as expressing inter-tissue energy transfer between the follicle and shaft. This transfer did not trigger $\mathrm{KFe}_{3}$ localized crystallization, thus supporting a teleportation phenomenon.

- BEMFs are shown to delay crystallization A human miniorgan is shown transferring BEMFs from the hair follicle to an isolated short hair shafts segment vertically placed at $1 \mathrm{~mm}$ distance.

- The delaying advance of a paramagnetic solution $\left(\mathrm{KFe}_{3}\right)$. This is quantitatively demonstrated in the time measured of an average of 4 minutes, only when BEMFs are a factor. Both the follicle and shaft display magneto reception properties exhibited by BEMFs induction. This mechanism has been described in birds [5].

- Further support of the crystallization delay had been documented in a separate experiments showing a stark slowing of crystallization, this time Potassium Ferrocyanide in solution mixed with very fine iron particles of 2000 nanometers in diameter or $\left(\mathrm{KFe}_{2} 2 \mathrm{~K}\right)$ was used. This phenomenon was documented in Panel 2. In that experiment the BEMFs had penetrated a $1 \mathrm{~mm}$ glass barrier [7]. 


\section{SUMMARY AND CONCLUSSION}

\subsection{THE HUMAN HAIR AS A BIOELECTROSENSORY MINIORGAN}

The findings herein presented support the hair to be described as a Bioelectrosensory miniorgan. Both the follicle and the shaft are theorized to have electro receptive molecules. When a freshly plucked hair is in a control SSP $\mathrm{KFe}_{3}$ preparation and allowed to evaporate, there are concentric periodic heavy crystallization semicircles (Fig 3). When in the same preparation, the follicle is fronted by a vertically placed isolated short shaft segment, the evidence show the follicular BEMFs bypassing the area between the follicle and the isolated shaft. The BEMFs are discharged by one end of the cut shaft (Panel 1), that these heavy concentric crystallization discharges mimic hair follicles in control experiments. The observed BEMFs bypassing mechanism could point at teleported BEMFs.

It was also demonstrated that BEMFs delay the crystallization advance of Ferro (Panel 2) and Ferri (Panel 1) cyanides in solution.

\subsection{ADDENDUM}

Suggested Methodology to Duplicate the Reported Findings CAUTION Avoid Failure to Centering Isolated Cut Shaft and Failure to Keep Distance from Follicle

To aid the reader in duplicating the experiments, care must be taken to center the isolated short hair shaft segment vertically away from the follicle at $\cong 1 \mathrm{~mm}$ lateral distance. Failure to centering is shown in Figure 4 below.

\section{Avoid Improper Centering of Isolated Cut Shaft Segment}

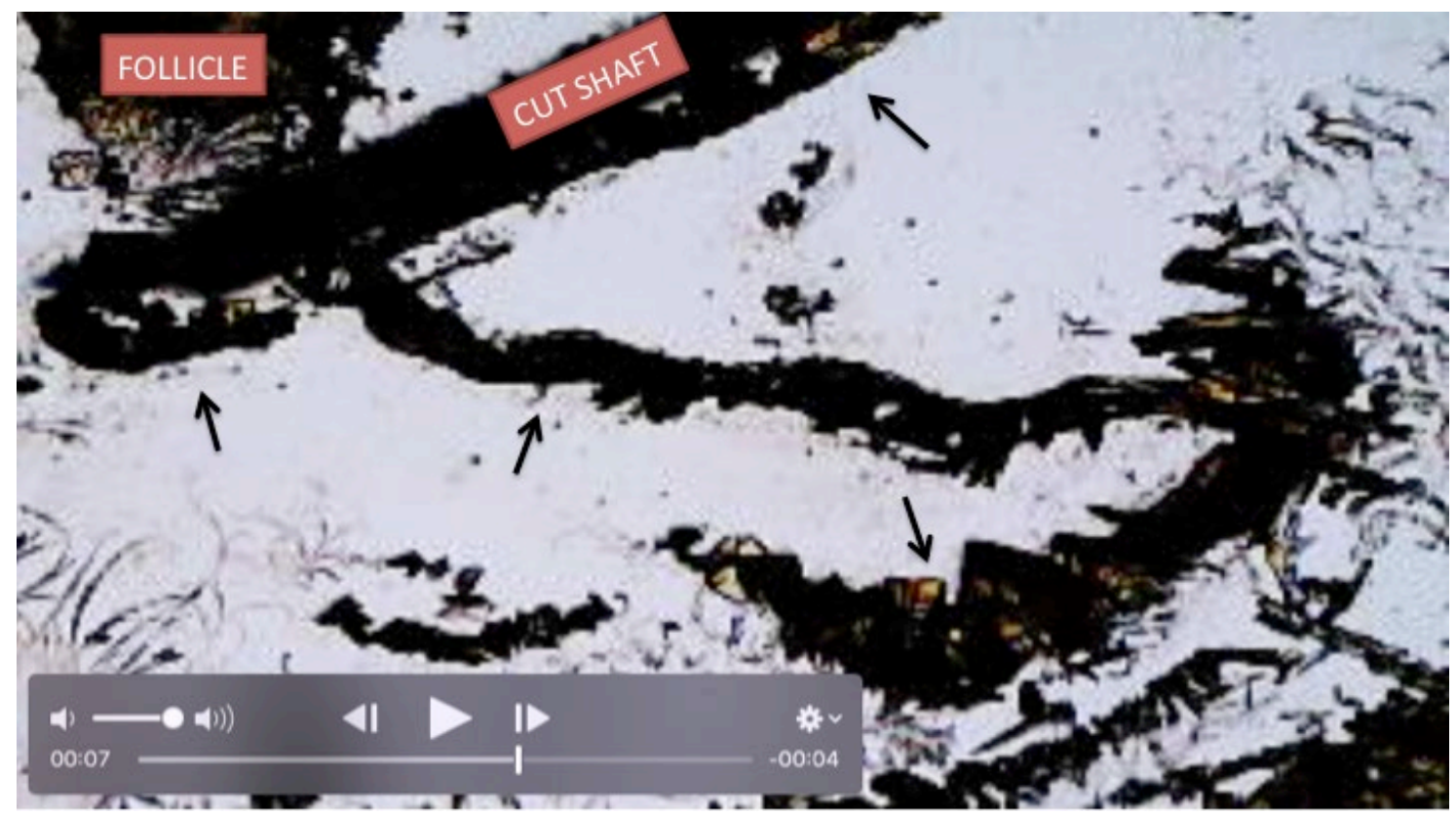

Figure 4: Cut shaft touching the follicle and non-center aligned. SSP KFe3 after evaporation- Black Arrows: Showing BEMFs energy dissipation bypassing misplaced obstacle (cut shaft); and triggering what appears to be electrical discharges. Please compare to Panel 1 and Figure 1 above.

\section{Avoid Having a Non-Vertical Contiguous Follicle, Long Isolated Shaft Connection.}

BEMFs appear to dissipate through the shaft (Figure 5).

The videorecording demonstrates an absence of heavy periodic crystallization exiting the shat. This observation is documented in the last few frames of videorecording. 


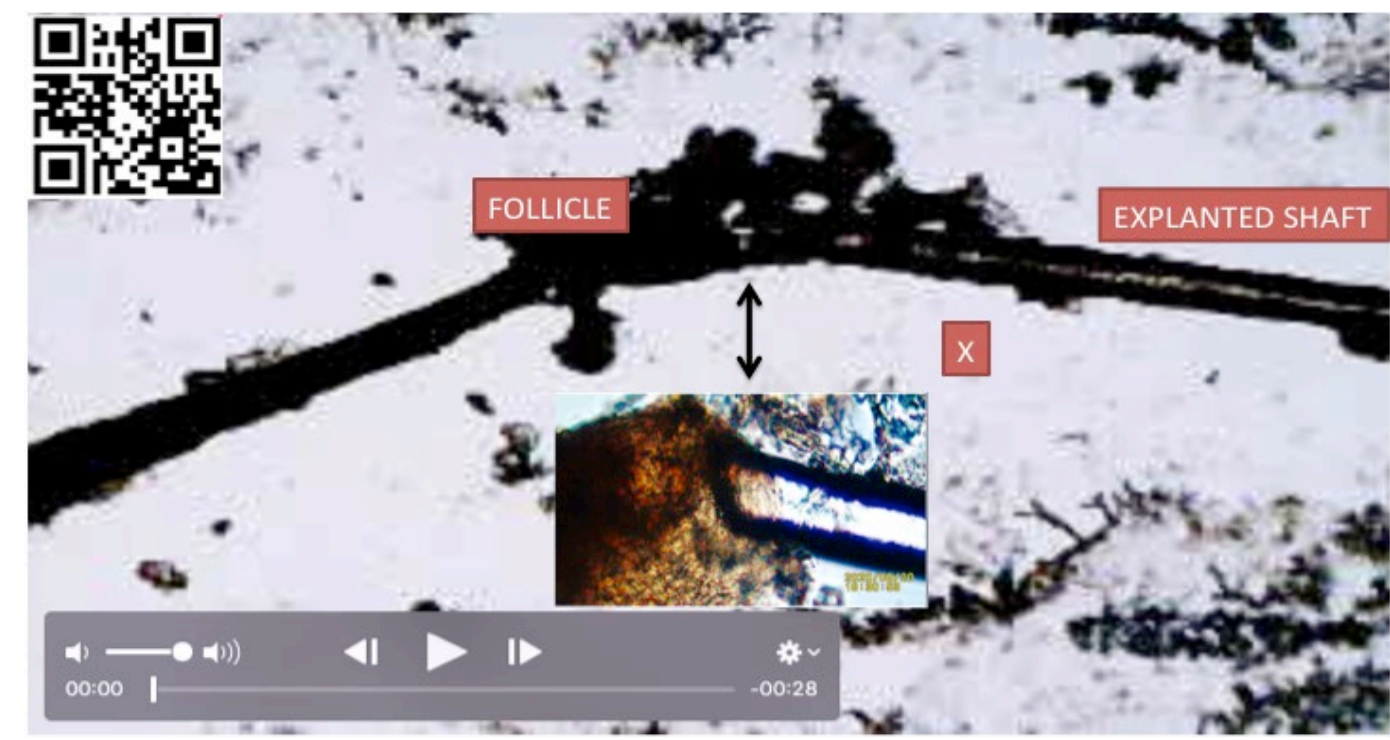

Figure 5: Showing disruption of concentric BEMFs waves by isolated cut shaft in contiguous (direct) contact with follicle. X: Area void of concentric crystallization.

Black Arrow: Pointing at amplified image of isolated shaft touching follicle.

For additional details link to: https://youtu.be/arcpZLkYqP0

Or scan QR Code in left upper corner of image.

\section{SOURCES OF FUNDING}

None.

\section{CONFLICT OF INTEREST}

None.

\section{ACKNOWLEDGMENT}

None.

\section{REFERENCES}

[1] Abrahám A. Embí. (2020). DEMONSTRATION OF THE HUMAN HAIR FOLLICLE MAGNETORECEPTION OF BIOMAGNETISM RADIATED BY THE CONCAVE PART OF THE HUMAN HAND. International Journal of Research -GRANTHAALAYAH, 8(5), 348 - 354. https://doi.org/10.29121/granthaalayah.v8.i5.2020.291

[2] Schneider, M. R., Schmidt-Ullrich, R., \& Paus, R. (2009). The hair follicle as a dynamic miniorgan. Current biology: $C B, 19$ (3), R132-R142. https://doi.org/10.1016/j.cub.2008.12.005

[3] D Cohen, Y Palti, B N Cuffin, S J Schmid (1980) Magnetic fields produced by steady currents in the body Proceedings of the National Academy of Sciences Mar 1980, 77 (3) 1447-1451; DOI:10.1073/pnas.77.3.1447

[4] Burnei, G., Hodorogea, D., Georgescu, I., Gavriliu, Ş., Drăghici, I., Dan, D., Vlad, C., \& Drăghici, L. (2012). The electromagnetic bio-field: clinical experiments and interferences. Journal of medicine and life, 5(2), 139-144.

[5] Embí AA, Jacobson JI, Sahoo K, Scherlag BJ (2015) Demonstration of Inherent Electromagnetic Energy Emanating from Isolated Human Hairs. Journal of Nature and Science, 1(3): e55.

[6] Abrahám A. Embí, Benjamin J. Scherlag (2015) Human hair follicle biomagnetism: potential biochemical correlates. Journal of Molecular Biochemistry 4, 32-35

[7] Abrahám A. Embí, Benjamin J. Scherlag. (2016) Demonstration of Human Hair Follicle Biomagnetic Penetration Through Glass Barriers. International Journal of Materials Chemistry and Physics Vol. 2, No. 2, pp. 71-74 